\title{
The McBurr XII and Log-McBurr XII Models with Applications to Lifetime Data
}

\author{
Gauss M. Cordeiro ${ }^{1}$, Edwin M. M. Ortega ${ }^{2}$, G. G. Hamedani ${ }^{3}$, Diogo A. Garcia ${ }^{4}$ \\ ${ }^{1}$ Departamento de Estatística, Universidade Federal de Pernambuco, Brazil \\ ${ }^{2}$ Departamento de Ciências Exatas, Universidade de São Paulo, Brazil \\ ${ }^{3}$ Department of Mathematics, Statistics and Computer Science, Marquette University, Milwaukee, USA \\ ${ }^{4}$ Departamento de Melhoramento Genético Animal, Universidade Estadual Paulista Júlio de Mesquita Filho, Brazil \\ Correspondence: Edwin M. M. Ortega, Departamento de Ciências Exatas, Universidade de São Paulo, Brazil. E-mail: \\ edwin@usp.br
}

Received: August 4, 2015 Accepted: September 3, 2015 Online Published: January 1, 2016

doi:10.5539/ijsp.v5n1p1 URL: http://dx.doi.org/10.5539/ijsp.v5n1p1

\begin{abstract}
A new six-parameter extended fatigue lifetime model named the McDonald-Burr XII distribution is introduced, which generalizes the Burr XII, beta Burr XII (Paranaíba et al., 2011) and Kumaraswamy-Burr XII (Paranaíba et al., 2012) distributions. The proposed distribution is characterized in terms of truncated moments. We obtain the ordinary and incomplete moments and quantile and generating functions. We estimate the model parameters by maximum likelihood. A Monte Carlo simulation is performed to study the asymptotic normality of the estimates. We also propose an extended regression model based on the logarithm of the McDonald-Burr XII distribution that can be more realistic in the analysis of real data than other special regression models. Two applications to real data validate the importance of the new models.
\end{abstract}

Keywords: Burr XII distribution, Kumaraswamy distribution, maximum likelihood estimation, McDonald distribution, regression model

\section{Introduction}

Continuous univariate distributions have been extensively used over the past decades for modeling data in several fields such as environmental and medical sciences, engineering, demography, biological studies, actuarial, economics, finance and insurance. However, in many applied areas such as lifetime analysis, finance and insurance, there is a clear need for extended forms of these distributions. The Burr XII (BXII) model is a widely used distribution for modeling lifetime data. One of its advantages is that the survival function has a closed-form expression. However, it does not provide a reasonable parametric fit for non-monotone failure rates such as the bathtub shaped and unimodal failure rates that are common in reliability and biological studies. Such bathtub hazard curves have nearly flat middle portions and the associated densities have a positive anti-mode. Unimodal failure rates can be observed in course of a disease whose mortality rate reaches a peak after some finite period and then declines gradually. More recently, there has been a great interest among statisticians and applied researchers in constructing flexible distributions for better modeling non-monotone failure rates.

The BXII distribution with shape parameters $k>0$ and $\alpha>0$ and scale parameter $s>0$ has probability density function (pdf) given by

$$
g(x ; s, k, \alpha)=\frac{k \alpha}{s^{\alpha}} x^{\alpha-1}\left[1+\left(\frac{x}{s}\right)^{\alpha}\right]^{-(k+1)}, \quad x>0 .
$$

If $\alpha>1$, the density function is unimodal with mode at $v=s[(\alpha-1) /(k \alpha+1)]^{1 / \alpha}$ and is L-shaped if $\alpha=1$. If $q<k \alpha$, the $q$ th moment about zero is $\mu_{q}^{\prime}=k s^{q} B\left(k-q \alpha^{-1}, 1+q \alpha^{-1}\right)$, where $B(a, b)=\int_{0}^{1} w^{a-1}(1-w)^{b-1} d w=\Gamma(a) \Gamma(b) / \Gamma(a+b)$ denotes the beta function for $a>0$ and $b>0$ and $\Gamma(a)=\int_{0}^{\infty} w^{a-1} \mathrm{e}^{-w} d w$ is the gamma function. The associated survival function to (1) is $S(x ; s, k, \alpha)=\left[1+(x / s)^{\alpha}\right]^{-k}$.

In this paper, we study some structural properties of the new six-parameter model called the Mc-Burr XII ("McBXII") distribution defined in the next section by equation (2).

The rest of the paper is outlined as follows. The McBXII distribution is formally defined in Section 2. In Sections 3 to 6, we provide some structural properties of the new model. In Section 7, we estimate the model parameters by maximum likelihood and present a simulation study to investigate the asymptotic normality of the maximum likelihood estimates (MLEs). In Section 8, we propose the $\log -M c B X I I$ ("LMcBXII") regression model for censoring data. In Section 9, we 
demonstrate empirically by means of two applications to real data the usefulness of the new models and provide a clear evidence that they yield better fits than some other known models. Section 10 ends with some concluding remarks.

\section{The McBXII Distribution}

The McBXII density function is given (for $x>0$ ) by

$$
\begin{aligned}
f_{\mathrm{McBXII}}(x)= & \frac{c k \alpha x^{\alpha-1}}{B(a, b) s^{\alpha}}\left[1+\left(\frac{x}{s}\right)^{\alpha}\right]^{(-k-1)}\left\{1-\left[1+\left(\frac{x}{s}\right)^{\alpha}\right]^{-k}\right\}^{a c-1} \times \\
& {\left[1-\left\{1-\left[1+\left(\frac{x}{s}\right)^{\alpha}\right]^{-k}\right\}^{c}\right]^{b-1}, }
\end{aligned}
$$

where $k, \alpha, a, b$ and $c$ are positive shape parameters and $s>0$ is a scale parameter. Hereafter, a random variable $X$ with pdf (2) is denoted by $X \sim \operatorname{McBXII}(a, b, c, s, k, \alpha)$. The survival function of $X$ is given by

$$
S_{\mathrm{McBXII}}(x)=1-I_{\left\{1-\left[1+\left(\frac{x}{s}\right)^{\alpha}\right]^{-k}\right\}^{c}}(a, b),
$$

where $I_{y}(a, b)=B_{y}(a, b) / B(a, b)$ is the incomplete beta function ratio, $B_{y}(a, b)=\int_{0}^{y} \omega^{a-1}(1-\omega)^{b-1} d \omega$ is the incomplete beta function, and $a>0, b>0$ and $c>0$ are additional shape parameters.

The hazard rate function (hrf) corresponding to (2) is given by

$$
\begin{aligned}
h_{\mathrm{McBXII}}(x)= & \frac{c k \alpha x^{\alpha-1}\left[1+\left(\frac{x}{s}\right)^{\alpha}\right]^{-(k+1)}\left\{1-\left[1+\left(\frac{x}{s}\right)^{\alpha}\right]^{-k}\right\}^{a c-1}}{s^{\alpha} B(a, b)\left[1-I_{\left.\left\{\left[1+\left(\frac{x}{s}\right)^{\alpha}\right]^{-k}\right\}^{c}(a, b)\right]}\right.} \times \\
& {\left[1-\left\{1-\left[1+\left(\frac{x}{s}\right)^{\alpha}\right]^{-k}\right\}^{c}\right]^{b-1} . }
\end{aligned}
$$

The study of the new model is important since it extends several distributions previously considered in the literature. In fact, the BXII model (with parameters $s, k$ and $\alpha$ ) is clearly a basic exemplar when $a=b=c=1$. The McBXII model contains as sub-models the beta-Burr XII (BBXII) (Paranaíba et al., 2011) and Kumaraswamy-Burr XII (KwBXII) (Paranaíba et al., 2012) distributions when $c=1$ and $a=1$, respectively. For $b=c=1$, it becomes the exponentiated Burr XII (EBXII) distribution with power parameter $a$, which has not been investigated. For $s=m^{-1}$ and $k=1$, it reduces to a new distribution referred to as the beta $\log$-logistic (BLL) distribution. For $a=b=c=1, s=m^{-1}$ and $k=1$, it gives the LL distribution. For $a=c=\alpha=1$ and $a=b=c=\alpha=1$, it reduces to the beta Pareto type II (BPII) and Pareto type II (PII) distributions, respectively. If $k \rightarrow \infty$, it is identical to the beta-Weibull (BW) distribution. If $k \rightarrow \infty$ in addition to $a=b=c=1$, it becomes the Weibull distribution.

Plots of the McBXII density and hazard rate functions for selected parameter values are displayed in Figures 1 and 2 , respectively. These functions can take various shapes depending on the parameter values. Figure 3 provides some relationships among some special models obtained from the McBXII distribution.

Let $Q_{a, b}(u)$ be the beta quantile function (qf) with positive parameters $a$ and $b$. The qf of $X$, say $x=Q(u)$, follows by inverting $S_{\mathrm{McBXII}}(x)$. It is expressed in terms of the BXII qf as $Q(u)=Q_{\mathrm{BXII}}\left(Q_{a, b}(u)^{1 / c}\right)$, where $Q_{\mathrm{BXII}}(u)=s[(1-$ $\left.\left.u^{1 / c}\right)^{-1 / k}-1\right]^{1 / \alpha}$.

\section{Expansion for the McBXII Density Function}

We provide some useful expansions for the McG density function based on the concept of exponentiated distribution. For an arbitrary baseline cdf $G(x)$, a random variable $Z$ is said to have the exponentiated-G ("exp-G" for short) distribution with power parameter $a>0$, say $Z \sim \exp -\mathrm{G}(a)$, if the cdf and pdf are given by $H_{a}(x)=G^{a}(x)$ and $h_{a}(x)=a G^{a-1}(x) g(x)$, respectively. The properties of exponentiated distributions have been studied by several authors. See, for example, Mudholkar et al. (1995) and Nadarajah and Gupta (2007) for exponentiated Weibull (EW) and exponentiated gamma distributions, respectively.

For brevity of notation, we shall drop the explicit reference to the parent parameters $\alpha, s$ and $k$ unless otherwise stated. Alexander et al. (2012) demonstrated that the McG density function can be expressed as a linear combination of exp-G densities given by

$$
f_{M c G}(x ; a, b, c)=\sum_{i=0}^{\infty} w_{i} h_{a(i+c)}(x)
$$


(a)

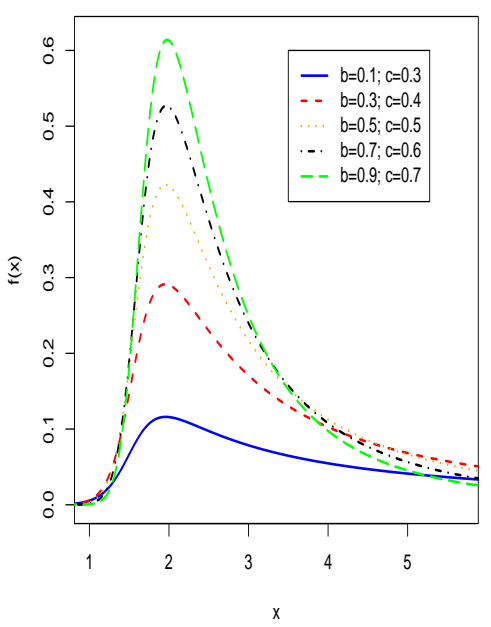

(b)

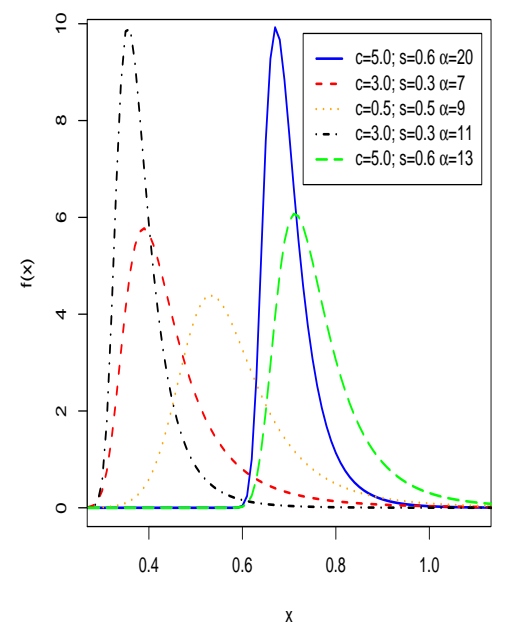

(c)

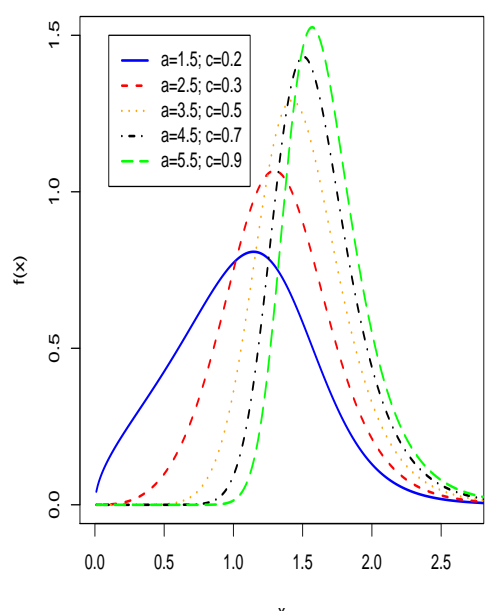

Figure 1. Plots of the McBXII density function. (a) For $a=2.5, s=1.6, k=0.3$ and $\alpha=9.5$. (b) For $a=3.5, b=0.5$ and $k=1.5$. (c) For $b=0.5, s=1.6, k=4.0$ and $\alpha=5.1$.

(a)

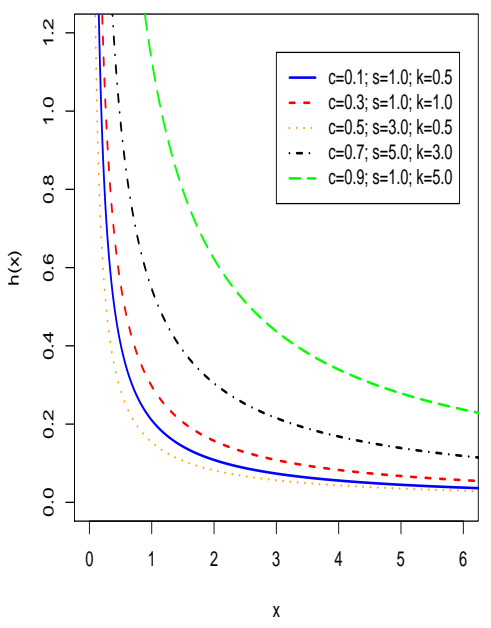

(b)

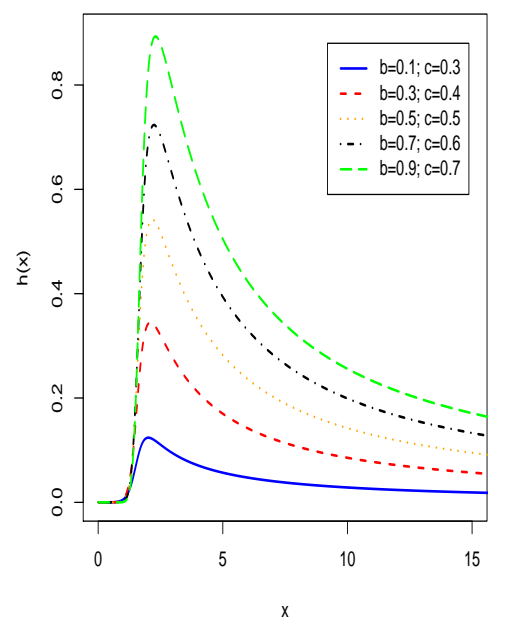

(c)

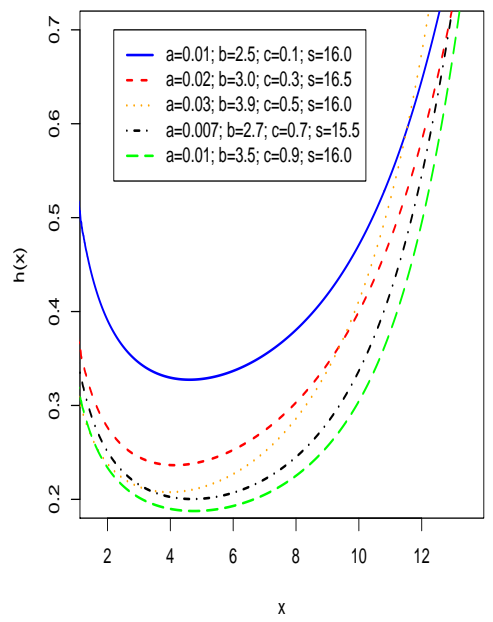

Figure 2. Plots of the McBXII hrf. (a) Decreasing: For $a=0.8, b=1.5$ and $\alpha=0.3$. (b) Unimodal: For $a=2.5, s=1.6$, $k=0.3$. (c) Bathtub: For $k=1.0$ and $\alpha=12.0$.

where $h_{a(i+c)}(x)$ denotes the exp-G density with power parameter $a(i+c)$ and

$$
w_{i}=\frac{(-1)^{i}}{(a+i) B(a, b+1)}\left(\begin{array}{l}
b \\
i
\end{array}\right) .
$$

Based on the mixture representation (5), we can derive certain McG properties from those properties of the exp-G distributions. Taking the BXII distribution defined in (1) for the baseline G, we can write the exp-BXII density function with power parameter $a(i+c)$, say exp-BXII $a(i+c)]$, as

$$
h_{a(i+c)}(x)=\frac{a(i+c) k \alpha}{s^{\alpha}} x^{\alpha-1}\left[1+\left(\frac{x}{s}\right)^{\alpha}\right]^{-(k+1)}\left\{1-\left[1+\left(\frac{x}{s}\right)^{\alpha}\right]^{-k}\right\}^{a(i+c)-1} .
$$




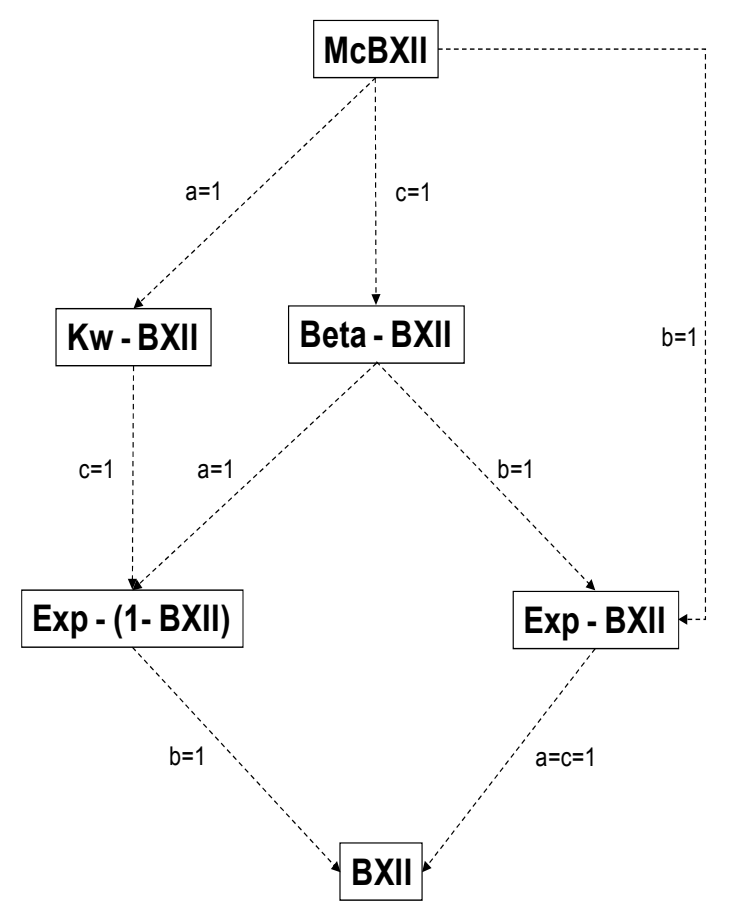

Figure 3. Relationships of the McBXII sub-models.

For any nonnegative integer $a$ and $|z|<1$, the power series $(1-z)^{a-1}=\sum_{j=0}^{\infty}\left(\begin{array}{c}a-1 \\ j\end{array}\right)(-z)^{j}$ holds. Using this expansion, we can write $h_{a(i+c)}(x)$ as

$$
h_{a(i+c)}(x)=\sum_{j=0}^{\infty} v_{j}^{(i)} g(t ; s,(j+1) k, \alpha),
$$

where

$$
v_{j}^{(i)}=\frac{(-1)^{j} a(i+c)}{(j+1)}\left(\begin{array}{c}
a(i+c)-1 \\
j
\end{array}\right)
$$

Combining equations (5) and (6), the McBXII density function can be expressed as

$$
f_{M c B X I I}(x ; a, b, c, s, k, \alpha)=\sum_{j=0}^{\infty} t_{j} g(x ; s,(j+1) k, \alpha),
$$

where

$$
t_{j}=t_{j}(a, b, c)=\frac{(-1)^{j} a}{(j+1) B(a, b+1)} \sum_{i=0}^{\infty} \frac{(-1)^{i}(c+i)}{(a+i)}\left(\begin{array}{l}
b \\
i
\end{array}\right)\left(\begin{array}{c}
a(c+i)-1 \\
j
\end{array}\right) .
$$

Equation (7) reveals that the McBXII density function is an infinite mixture of BXII density functions. So, several McBXII mathematical properties can be obtained from those of the BXII distribution.

\section{Moments}

For $n<k \alpha$, the $n$th moment of $X$ follows from (7) using a result given in Section 1:

$$
\mu_{n}^{\prime}=E\left(X^{n}\right)=k s^{n} \sum_{j=0}^{\infty}(j+1) t_{j} B\left((j+1) k-n \alpha^{-1}, 1+n \alpha^{-1}\right) .
$$

When the condition $n<k \alpha$ does not hold, we can consider the skewness and kurtosis measures based on quantiles. The Bowley skewness (see Kenney and Keeping, 1962) is based on quartiles $B=[Q(3 / 4)-2 Q(1 / 2)+Q(1 / 4)] /[Q(3 / 4)-$ $Q(1 / 4)]$, whereas the Moors kurtosis (see Moors, 1998) is based on octiles $M=[Q(7 / 8)-Q(5 / 8)-Q(3 / 8)+Q(1 / 8)] /[Q(6 / 8)-$ $Q(2 / 8)$ ], where $Q(\cdot)$ denotes the McBXII qf given in Section 2. We investigate the effect of the shape parameter $c$ on these measures and conclude that this parameter acts primarily as another skewness parameter rather than a kurtosis parameter. 


\section{Characterization Results Based on Two Truncated Moments}

In practice, an investigator will be usually interested to know if the model under study fits the requirements of the proposed distribution. To this end, the investigator will rely on the characterizations of the selected distribution. Generally speaking, the problem of characterizing a distribution is an important problem in various fields and has recently attracted the attention of many researchers. Consequently, various characterization results have been reported in the literature.

Our characterization follows a result (Glnzel, 1987) given in Theorem B.1 of Appendix B.

Proposition 1. Let $X: \Omega \rightarrow(0, \infty)$ be a continuous random variable and let $h(x)=\left\{1-\left[1+(x / s)^{\alpha}\right]^{-k}\right\}^{1-a c}$ and $q(x)=x^{\alpha}\left\{1-\left[1+(x / s)^{\alpha}\right]^{-k}\right\}^{1-a c}$ for $x \in(0, \infty)$. The pdf of $X$ for $b=1$ and $k>1$ is (2) if and only if the function $\eta$ defined in Theorem B.1 is given by

$$
\eta(x)=\frac{s^{\alpha}}{(k-1)}\left[1+k\left(\frac{x}{s}\right)^{\alpha}\right], x>0 .
$$

Proof. If $X$ has pdf (2), then

$$
\left[1-F_{M c B X I I}(x)\right] \mathbf{E}[h(X) \mid X \geq x]=a c\left[1+\left(\frac{x}{s}\right)^{\alpha}\right]^{-k}, \quad x>0,
$$

and

$$
\left[1-F_{M c B X I I}(x)\right] \mathbf{E}[q(X) \mid X \geq x]=a c\left[1+\left(\frac{x}{s}\right)^{\alpha}\right]^{-k}\left\{\frac{s^{\alpha}}{(k-1)}\left[1+k\left(\frac{x}{s}\right)^{\alpha}\right]\right\}, \quad x>0 .
$$

Finally,

$$
\eta(x) h(x)-q(x)=\left\{1-\left[1+\left(\frac{x}{s}\right)^{\alpha}\right]^{-k}\right\}^{1-a c}\left(\frac{x^{\alpha}+s^{\alpha}}{k-1}\right)>0, \quad \text { for } k>1 \text { and } x>0 .
$$

Conversely, if $\eta$ is given as above, then $s^{\prime}(x)=k \alpha x^{\alpha-1} /\left(x^{\alpha}+s^{\alpha}\right)$ and hence $s(x)=k \log \left(x^{\alpha}+s^{\alpha}\right)+C_{1}, x>0$, where $C_{1}$ is a constant. Now, in view of Theorem B.1 (with $C$ chosen appropriately), $X$ has pdf (2). Clearly, there are other triplets $(h, q, \eta)$ satisfying the conditions of Proposition 1.

Corollary 1. Let $h(x)=\left\{1-\left[1+(x / s)^{\alpha}\right]^{-k}\right\}^{1-a c}$ for $x \in(0, \infty)$ and let $X: \Omega \rightarrow(0, \infty)$ be a continuous random variable. The pdf of $X$ for $k>1$ is (2) if and only if there exist functions $q$ and $\eta$ defined in Theorem B.1 satisfying the differential equation

$$
\frac{\eta^{\prime}(x)\left\{1-\left[1+\left(\frac{x}{s}\right)^{\alpha}\right]^{-k}\right\}^{1-a c}}{\eta(x)\left\{1-\left[1+\left(\frac{x}{s}\right)^{\alpha}\right]^{-k}\right\}^{1-a c}-q(x)}=\frac{k \alpha x^{\alpha-1}}{x^{\alpha}+s^{\alpha}}, \quad x>0 .
$$

The general solution of the differential equation given in Corollary 1 is

$$
\eta(x)=\left(x^{\alpha}+s^{\alpha}\right)^{k}\left[-k \alpha \int q(x) x^{\alpha-1}\left(x^{\alpha}+s^{\alpha}\right)^{-k-1}\left\{1-\left[1+\left(\frac{x}{s}\right)^{\alpha}\right]^{-k}\right\}^{a c-1} d x+D\right],
$$

for $x>0$, where $D$ is a constant.

\section{Generating Function}

Let $M_{k}(t)$ be the $\operatorname{BXII}(s, k, \alpha)$ generating function. The moment generating function (mgf) $M(t)$ of $X$ can be obtained from equation (7) as

$$
M(t)=\sum_{j=0}^{\infty} t_{j} M_{(j+1) k}(t),
$$

where $M_{(j+1) k}(t)$ is the $\operatorname{mgf}$ of the $\operatorname{BXII}(s,(j+1) k, \alpha)$ distribution.

If we assume $\alpha=m / r$, where $m$ and $r$ are positive integers, we can provide a simple representation for $M_{k}(t)$ (for $t<0$ ) as

$$
M_{k}(t)=\frac{k m}{r} \int_{0}^{\infty} \mathrm{e}^{\mathrm{stx}} \mathrm{x}^{\mathrm{m} / \mathrm{r}-1}\left(1+\mathrm{x}^{\mathrm{m} / \mathrm{r}}\right)^{-(\mathrm{k}+1)} \mathrm{dx}=\frac{\mathrm{km}}{\mathrm{r}} \mathrm{I}\left(-\mathrm{st}, \frac{\mathrm{m}}{\mathrm{r}}+1, \frac{\mathrm{m}}{\mathrm{r}},-\mathrm{k}-1\right) .
$$

The $I(\cdot)$ function can be expressed in terms of the Meijer G-function (Gradshteyn and Ryzhik, 2000) and evaluated in Mathematica software. The condition $\alpha=m / r$ is not restrictive since every positive real number can be approximated by a rational number. 


\section{Maximum Likelihood Estimation}

Let $X_{i}$ be a random variable following (2) with the vector $\boldsymbol{\theta}=(a, b, c, s, k, \alpha)^{T}$ of parameters. The data encountered in survival analysis and reliability studies are often censored. Parametric inference for such data is usually based on likelihood methods and asymptotic theory.

Consider a sample $\left(x_{1}, \ldots, x_{n}\right)$ of $n$ independent observations, where each random response is defined by $x_{i}=\min \left\{X_{i}, D_{i}\right\}$ and each individual $i$ is assumed to have a lifetime $X_{i}$ and a censoring time $D_{i}$. We assume non-informative censoring such that the observed lifetimes and censoring times are independent. Let $F$ and $D$ be the sets of individuals for which $x_{i}$ is the lifetime and censoring, respectively. The log-likelihood function for the vector of parameters $\boldsymbol{\theta}$ from model (2) has the form $l(\boldsymbol{\theta})=\sum_{i \in F} \log \left[f_{\mathrm{McBXII}}\left(x_{i}\right)\right]+\sum_{i \in D} \log \left[S_{\text {McBXII }}\left(x_{i}\right)\right]$, where $f_{\mathrm{McBXII}}\left(x_{i}\right)$ is the density function (2) and $S_{\mathrm{McBXII}}\left(x_{i}\right)$ is the survival function (3) of $X_{i}$.

The censored log-likelihood $l(\boldsymbol{\theta})$ for the model parameters is

$$
\begin{aligned}
l(\boldsymbol{\theta})= & r[\log (c)+\log (\alpha)+\log (k)-\alpha \log (s)]-r \log [B(a, b)]+\left(\frac{k+1}{k}\right) \sum_{i \in F} \log \left(1-u_{i}\right)+ \\
& (\alpha-1) \sum_{i \in F} \log \left(x_{i}\right)+(a c-1) \sum_{i \in F} \log \left(u_{i}\right)+(b-1) \sum_{i \in F} \log \left(1-u_{i}^{c}\right)+\sum_{i \in D} \log \left[1-I_{u_{i}^{c}}(a, b)\right],
\end{aligned}
$$

where $u_{i}=1-\left[1+\left(\frac{x_{i}}{s}\right)^{\alpha}\right]^{-k}$ and $r$ is the number of failures.

The score functions $U_{a}(\boldsymbol{\theta}), U_{b}(\boldsymbol{\theta}), U_{c}(\boldsymbol{\theta}), U_{s}(\boldsymbol{\theta}), U_{k}(\boldsymbol{\theta})$ and $U_{\alpha}(\boldsymbol{\theta})$ for the parameters $a, b, c, s, k$ and $\alpha$, respectively, are given in Appendix A.

The MLE $\widehat{\boldsymbol{\theta}}$ of $\boldsymbol{\theta}$ is obtained by solving the nonlinear equations $U_{a}(\boldsymbol{\theta})=0, U_{b}(\boldsymbol{\theta})=0, U_{c}(\boldsymbol{\theta})=0, U_{s}(\boldsymbol{\theta})=0, U_{k}(\boldsymbol{\theta})=0$ and $U_{\alpha}(\boldsymbol{\theta})=0$. These equations cannot be solved analytically and statistical software can be used to evaluate the estimates numerically. We can use iterative techniques such as a Newton-Raphson type algorithm to calculate $\widehat{\boldsymbol{\theta}}$. Initial values for the parameters can be obtained from the fitted McBXII special models. The corresponding SAS code is given in Appendix C.

For interval estimation of $(a, b, c, s, k, \alpha)$ and hypothesis tests on these parameters, we can obtain the observed information matrix since its expectation requires numerical integration. The elements of the $6 \times 6$ observed information matrix $J(\theta)=$ $\left\{-J_{r s}\right\}$ (for $\left.r, s=a, b, c, s, k, \alpha\right)$ can be requested from the authors. Under conditions that are fulfilled for parameters in the interior of the parameter space but not on the boundary, the multivariate normal $N_{6}\left(\mathbf{0}, J(\widehat{\boldsymbol{\theta}})^{-1}\right)$ distribution can be used to construct approximate confidence intervals for the parameters.

We can compute the maximized unrestricted and restricted log-likelihoods to obtain likelihood ratio (LR) statistics for testing some McBXII special models. For example, we can adopt LR statistics to check if the fitted McBXII distribution for a given data set is statistically "superior" to the fitted BBXII, KwBXII, BW, EBXII and BLL distributions.

\subsection{Simulations Study}

To examine the performance of the McBXII distribution, various simulations are performed for different sample sizes. The simulations are performed as follow:

- The data are generated from $x=u^{1 / \alpha} / s$, where $u=\left[\left(1-v^{1 / c}\right)^{-(1 / k)}\right]-1$ and $v \sim \operatorname{Beta}(a, b)$.

- The parameter values are set at $a=2.5, b=0.5, c=0.3, s=0.4, k=2.2$ and $\alpha=5.0$.

- The sample sizes are $n=100, n=300$ and $n=500$.

- Each sample size is replicated 1,000 times.

From the results of the simulations given in Table 1, we can verify that the root mean squared errors (RMSEs) of the MLEs of $a, b, c, s, k$ and $\alpha$ decay toward zero as the sample size increases, as usually expected under standard regularity conditions. As the sample size $n$ increases, the mean estimates of the parameters tend to be closer to the true parameter values. This fact supports that the asymptotic normal distribution provides an adequate approximation to the finite sample distribution of the estimates. The usual normal approximation can be oftentimes improved by making bias adjustments to the MLEs. Approximations to the biases of the MLEs in simple models may be obtained analytically. Bias correction typically does a very good job in reducing the bias. However, it may increase the mean squared error. Whether bias correction is useful in practice depends basically on the shape of the bias function and on the variance of the MLE. In order to improve the accuracy of MLEs using analytical bias reduction, one needs to obtain several cumulants of log likelihood derivatives, which are notoriously cumbersome for the proposed model. 
Table 1. Monte Carlo simulation results: mean estimates and RMSEs of $\hat{a}, \hat{b}, \hat{c}, \hat{s}, \hat{k}$ and $\hat{\alpha}$.

\begin{tabular}{lrrr}
\hline $\mathrm{n}$ & Parameter & \multicolumn{1}{c}{ Mean } & \multicolumn{1}{c}{ RMSE } \\
\hline \multirow{4}{*}{100} & $a$ & 2.323 & 0.626 \\
& $b$ & 0.683 & 0.141 \\
& $c$ & 0.343 & 0.047 \\
& $s$ & 0.436 & 0.003 \\
& $k$ & 2.684 & 2.014 \\
300 & $\alpha$ & 5.331 & 0.899 \\
\hline \multirow{6}{*}{500} & $a$ & 2.387 & 0.606 \\
& $b$ & 0.651 & 0.129 \\
& $c$ & 0.324 & 0.045 \\
& $s$ & 0.420 & 0.002 \\
& $\alpha$ & 2.475 & 1.857 \\
& $\alpha$ & 5.249 & 0.563 \\
\hline \multirow{6}{*}{5} & $a$ & 2.482 & 0.573 \\
& $b$ & 0.537 & 0.118 \\
& $c$ & 0.319 & 0.036 \\
\hline \hline
\end{tabular}

\section{The Log-McBurr XII Regression Model}

Based on the random variable $X$ following the McBXII density function (2), we define the random variable $Y=\log (X)$ having the LMcBXII density (for $-\infty<y<\infty$ ), which parameterized in terms of $\sigma=\alpha^{-1}$ and $\mu=\log (s)$, becomes

$$
\begin{aligned}
f(y)= & \frac{c k}{\sigma B(a, b)} \exp \left(\frac{y-\mu}{\sigma}\right)\left[1+\exp \left(\frac{y-\mu}{\sigma}\right)\right]^{-k-1}\left\{1-\left[1+\exp \left(\frac{y-\mu}{\sigma}\right)\right]^{-k}\right\}^{a c-1} \times \\
& {\left[1-\left\{1-\left[1+\exp \left(\frac{y-\mu}{\sigma}\right)\right]^{-k}\right\}^{c}\right]^{b-1}, }
\end{aligned}
$$

where $a, b, c, \sigma$ and $k$ are positive parameters and $-\infty<\mu<\infty$. The random variable $Y$ having pdf (11) is denoted by $Y \sim \operatorname{LMcBXII}(a, b, c, k, \sigma, \mu)$, where $\mu$ is the location parameter, $\sigma>0$ is the scale parameter and $a, b, c$ and $k$ are positive shape parameters. Thus,

$$
\text { if } \quad X \sim \operatorname{McBXII}(a, b, c, s, k, \alpha) \quad \text { then } \quad Y=\log (X) \sim \operatorname{LMcBXII}(a, b, c, k, \sigma, \mu) .
$$

The LMcBXII distribution contains as special models some well-known distributions. It simplifies to the log-Burr XII (LBXII) distribution when $a=b=c=1$. If $k=c=1$, it reduces to the beta-logistic (BL) distribution. If $b=c=1$, it leads to the log-exponentiated Burr XII (LEBXII) distribution. Further, if $a=b=c=1$, in addition to $k=1$, it becomes the logistic distribution. If $a=1$, it gives the log-Kumaraswamy Burr XII (LKwBXII) distribution. Figure 4 displays the density function of $Y$ for selected parameter values. These plots indicate that the LMcBXII distribution could be a very flexible distribution for modeling the kurtosis of the data.

The corresponding survival function is given by

$$
S(y)=1-I_{\left\{1-\left[1+\exp \left(\frac{y-\mu}{\sigma}\right)\right]^{-k}\right\}^{c}}(a, b) .
$$

We define the standardized random variable $Z=(Y-\mu) / \sigma$ having density function

$$
\begin{aligned}
\pi(z ; a, b, c, k)= & \frac{c k \exp (z)}{B(a, b)}[1+\exp (z)]^{-k-1}\left\{1-[1+\exp (z)]^{-k}\right\}^{a c-1} \times \\
& {\left[1-\left\{1-[1+\exp (z)]^{-k}\right\}^{c}\right]^{b-1}, \quad-\infty<z<\infty . }
\end{aligned}
$$

The special case $a=b=c=1$ refers to the LBXII standard distribution. 
(a)

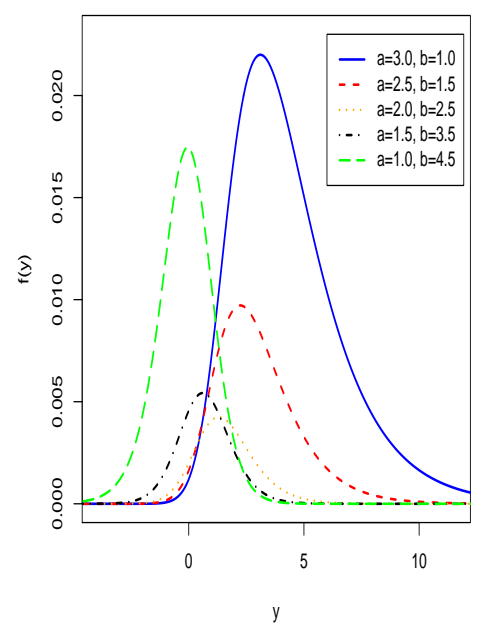

(b)

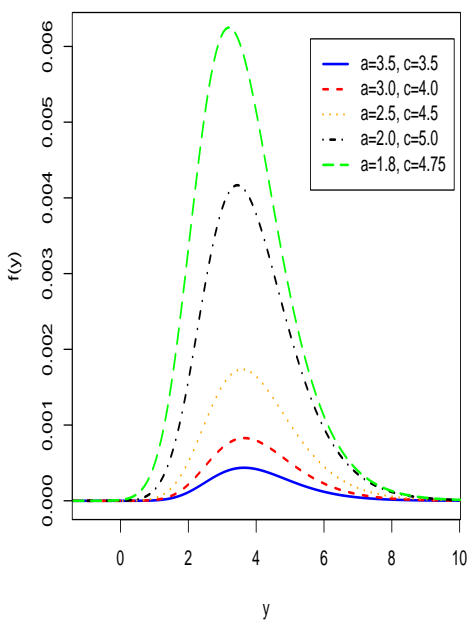

(c)

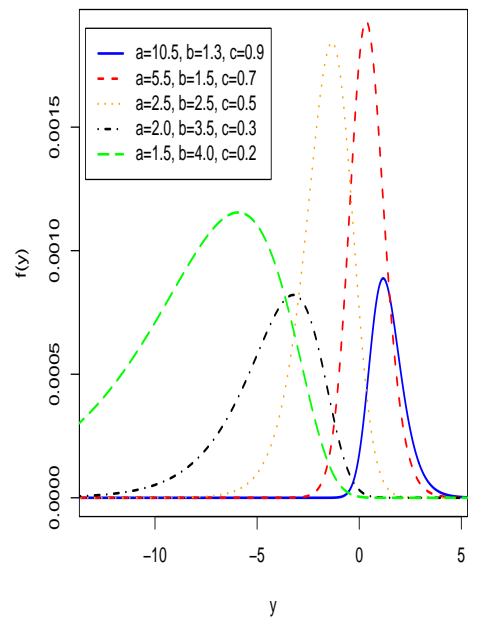

Figure 4. The LMcBXII density function. (a) For different values of $a$ and $b$ with $c=1.5, k=0.5, \mu=0$ and $\sigma=1$. (b) For different values of $a$ and $c$ with $b=2.5, k=0.5, \mu=0$ and $\sigma=1$. (c) For different values of $a, b$ and $c$ with $k=1.5$, $\mu=0$ and $\sigma=1$.

Parametric models to estimate univariate survival functions and for censored data regression problems are widely used. A parametric model that provides a good fit to lifetime data tends to yield more precise estimates of the quantities of interest. Based on the LMcBXII density, we propose a linear location-scale regression model or log-linear regression model linking the response variable $y_{i}$ and the explanatory variable vector $\mathbf{v}_{i}^{T}=\left(v_{i 1}, \ldots, v_{i p}\right)$ as follows

$$
y_{i}=\mathbf{v}_{i}^{T} \boldsymbol{\beta}+\sigma z_{i}, \quad i=1, \ldots, n,
$$

where the random error $z_{i}$ has density function (13), $\beta=\left(\beta_{1}, \ldots, \beta_{p}\right)^{T}, \sigma, a, b, c$ and $k$ are unknown parameters. The parameter $\mu_{i}=\mathbf{v}_{i}^{T} \boldsymbol{\beta}$ is the location of $y_{i}$. The location parameter vector $\boldsymbol{\mu}=\left(\mu_{1}, \ldots, \mu_{n}\right)^{T}$ is represented by a linear model $\boldsymbol{\mu}=\mathbf{V} \boldsymbol{\beta}$, where $\mathbf{V}=\left(\mathbf{v}_{1}, \ldots, \mathbf{v}_{n}\right)^{T}$ is a known model matrix. The model (14) is referred to as the LMcBXII regression model for censored data. It is an extension of an accelerated failure time model using the McBXII distribution for censored data and it opens new possibilities for fitting many different types of data.

Consider a sample $\left(y_{1}, \mathbf{v}_{1}\right), \ldots,\left(y_{n}, \mathbf{v}_{n}\right)$ of $n$ independent observations, where each random response is defined by $y_{i}=$ $\min \left\{\log \left(X_{i}\right), \log \left(D_{i}\right)\right\}$. We assume non-informative censoring such that the observed lifetimes and censoring times are independent. Let $F$ and $D$ be the sets of individuals for which $y_{i}$ is the log-lifetime or log-censoring, respectively. Conventional likelihood estimation techniques can be applied here. The log-likelihood function for the vector of parameters $\boldsymbol{\theta}=\left(a, b, c, k, \sigma, \boldsymbol{\beta}^{T}\right)^{T}$ from model (14) has the form $l(\boldsymbol{\theta})=\sum_{i \in F} l_{i}(\boldsymbol{\theta})+\sum_{i \in D} l_{i}^{(d)}(\boldsymbol{\theta})$, where $l_{i}(\boldsymbol{\theta})=\log \left[f\left(y_{i} \mid \mathbf{v}_{i}\right)\right]$, $l_{i}^{(d)}(\boldsymbol{\theta})=\log \left[S\left(y_{i} \mid \mathbf{v}_{i}\right)\right], f\left(y_{i} \mid \mathbf{v}_{i}\right)$ is the density (11) and $S\left(y_{i} \mid \mathbf{v}_{i}\right)$ is the survival function (12) of $Y_{i}$. The total log-likelihood function for $\boldsymbol{\theta}$ reduces to

$$
\begin{aligned}
l(\boldsymbol{\theta})= & r \log \left[\frac{c k}{\sigma B(a, b)}\right]-(k+1) \sum_{i \in F} \log \left[1+\exp \left(z_{i}\right)\right]+(a c-1) \sum_{i \in F} \log \left\{1-\left[1+\exp \left(z_{i}\right)\right]^{-k}\right\}+ \\
& \sum_{i \in F} z_{i}+(b-1) \sum_{i \in F} \log \left[1-\left\{1-\left[1+\exp \left(z_{i}\right)\right]^{-k}\right\}^{c}\right]+\sum_{i \in D} \log \left[1-I_{\left\{G\left(y_{i} \mid \mathbf{v}_{i}\right)\right\}^{c}}(a, b)\right],
\end{aligned}
$$

where $G\left(y_{i} \mid \mathbf{v}_{i}\right)=1-\left[1+\exp \left(\frac{y_{i}-\mathbf{v}_{i}^{T} \boldsymbol{\beta}}{\sigma}\right)\right]^{-k}, z_{i}=\left(y_{i}-\mathbf{v}_{i}^{T} \boldsymbol{\beta}\right) / \sigma$, and $r$ is the number of uncensored observations (failures).

The MLE $\hat{\boldsymbol{\theta}}$ of the parameter vector $\boldsymbol{\theta}$ of the LMcBXII regression model can be obtained by maximizing the log-likelihood function (15). The estimation process is also straightforward using the matrix programming language Ox (MAXBFGS subroutine) (see Doornik (2007)) or the procedure NLMixed in SAS. Initial values for $k, \sigma$ and $\boldsymbol{\beta}$ can be taken from the fit of the LBXII regression model with $a=b=c=1$. The NLMixed subroutine has been exhaustively used to estimate the model parameters for several distributions published in recent years. For example, Molenberghs et al. (2010) adopted this 
procedure to obtain the estimates in generalized linear models for repeated measures with normal and conjugate random effects, whereas Vangeneugden et al. (2011) used it to calculate the estimates of an extended random-effects model for repeated and overdispersed counts.

Initial values for $\beta$ and $\sigma$ are taken from the fit of the LBXII regression model with $a=b=1$ (see, Silva et al., 2008).

The asymptotic covariance matrix $K(\boldsymbol{\theta})^{-1}$ of $\widehat{\boldsymbol{\theta}}$ can be approximated by the inverse of the $(p+5) \times(p+5)$ observed information matrix $J(\boldsymbol{\theta})$, which is a consistent estimator for the asymptotic covariance matrix (Mudholkar et al., 1996). The multivariate normal distribution $N_{p+5}\left(0, J(\boldsymbol{\theta})^{-1}\right)$ for $\widehat{\boldsymbol{\theta}}$ can be used in the classical way to construct approximate confidence intervals for the parameters in $\boldsymbol{\theta}$.

We can use LR statistics for comparing some special models with the LMcBXII model. We consider the partition $\boldsymbol{\theta}=$ $\left(\boldsymbol{\theta}_{1}^{T}, \boldsymbol{\theta}_{2}^{T}\right)^{T}$, where $\boldsymbol{\theta}_{1}$ is a subset of parameters of interest and $\boldsymbol{\theta}_{2}$ is a subset of nuisance parameters. The LR statistic for testing the null hypothesis $H_{0}: \boldsymbol{\theta}_{1}=\boldsymbol{\theta}_{1}^{(0)}$ versus the alternative hypothesis $H_{1}: \boldsymbol{\theta}_{1} \neq \boldsymbol{\theta}_{1}^{(0)}$ is given by $w=2\{\ell(\widehat{\boldsymbol{\theta}})-\ell(\widetilde{\boldsymbol{\theta}})\}$, where $\widetilde{\boldsymbol{\theta}}$ and $\widehat{\boldsymbol{\theta}}$ are the estimates under the null and alternative hypotheses, respectively. The statistic $w$ is asymptotically (as $n \rightarrow \infty$ ) distributed as $\chi_{v}^{2}$, where $v$ is the dimension of the subset $\boldsymbol{\theta}_{1}$ of parameters of interest.

\subsection{Residual Analysis}

For studying departures from error assumptions as well as the presence of outliers, we consider two types of residuals: a deviance component residual (McCullagh and Nelder, 1989) and a martingale-type residual (Therneau et al., 1990). Therneau et al. (1990) pioneered the deviance component residual in counting process by using basically martingale residuals. The martingale residuals are skew, have maximum value +1 and minimum value $-\infty$. In parametric lifetime models, the martingale residual can be expressed as $r_{M_{i}}=\delta_{i}+\log \left[S\left(y_{i} ; \hat{\boldsymbol{\theta}}\right)\right]$, where $\delta_{i}=0$ if the $i$ th observation is censored and $\delta_{i}=1$ if the $i$ th observation is uncensored (see, for example, Klein and Moeschberger, 1997; Ortega et al., 2008; Silva et al., 2011). Hence, the martingale residuals for the LMcBXII model can be expressed as

$$
r_{M_{i}}= \begin{cases}1+\log \left\{1-I_{\left.\left[\widehat{G}\left(y_{i} \mid \mathbf{v}_{i}\right)\right]^{\hat{c}}(\hat{a}, \hat{b})\right\}}\right. & \text { if } \quad i \in F, \\ \log \left\{1-I_{\left.\left[\widehat{G}\left(y_{i} \mid \mathbf{v}_{i}\right)\right]^{\hat{c}}(\hat{a}, \hat{b})\right\}}\right. & \text { if } \quad i \in D,\end{cases}
$$

where the sets $F$ and $C$ and the $I(\cdot)$ function are defined in Section 8 and

$$
\widehat{G}\left(y_{i} \mid \mathbf{v}_{i}\right)=1-\left[1+\exp \left(\frac{y_{i}-\mathbf{v}_{i}^{T} \hat{\boldsymbol{\beta}}}{\hat{\sigma}}\right)\right]^{-\hat{k}} \text {. }
$$

The deviance component residual proposed by Therneau et al. (1990) is a transformation of the martingale residual to attenuate the skewness which was motivated by the deviance component residual in generalized linear models. In particular, the deviance component residuals for the Cox's proportional hazards model with time-dependent explanatory variables is given by

$$
r_{D_{i}}=\operatorname{sgn}\left(\mathrm{r}_{\mathrm{M}_{\mathrm{i}}}\right)\left\{-2\left[\mathrm{r}_{\mathrm{M}_{\mathrm{i}}}+\delta_{\mathrm{i}} \log \left(\delta_{\mathrm{i}}-\mathrm{r}_{\mathrm{M}_{\mathrm{i}}}\right)\right]\right\}^{1 / 2},
$$

where $\operatorname{sgn}(\cdot)$ is the sign function and $r_{M_{i}}$ is the martingale residual. Ortega et al. (2008) and Hashimoto et al. (2010) investigated the empirical distributions of $r_{M_{i}}$ and $r_{D_{i}}$ for the generalized log-gamma and LEW regression models for interval-censored data varying the sample sizes and censoring proportions, respectively.

Recently, Silva et al. (2011) proposed the modified martingale-type residual $\left(r_{M D_{i}}\right)$ for the BXII regression model with censored data. They are obtained by subtracting their expected values from the martingale-type residuals, which (based on the simulation studies) are approximately equal to 0.34 . So, the modified martingale-type residuals for the LMcBXII regression model with censored data are given by

$$
r_{M D_{i}}= \begin{cases}\operatorname{sign}\left\{1+\log \left[I_{\hat{q}_{i}^{\hat{c}}}(\hat{a}, \hat{b})\right]\right\} \times \\ \left\{-2\left[1+\log \left(1-I_{\hat{q}_{i}^{\hat{c}}}(\hat{a}, \hat{b})\right)+\log \left\{\log \left(1-I_{\hat{q}_{i}^{\hat{c}}}(\hat{a}, \hat{b})\right)\right\}\right]\right\}^{1 / 2}-0.34 & \text { if } \quad i \in F, \\ \operatorname{sign}\left\{\log \left[I_{\hat{q}_{i}^{\hat{c}}}(\hat{a}, \hat{b})\right]\right\}\left\{-2\left[\log \left(1-I_{\hat{q}_{i}^{\hat{c}}}(\hat{a}, \hat{b})\right]\right\}^{1 / 2},\right. & \text { if } \quad i \in D,\end{cases}
$$

where $\hat{q}_{i}=\widehat{G}\left(y_{i} \mid \mathbf{v}_{i}\right)$ is given by (16).

\section{Data Analysis}

In this section, we illustrate the usefulness of the McBXII and LMcBXII distributions by means of two real data sets. In the first and second applications, the sample sizes are $n=202$ and $n=1,326$, respectively. For these samples sizes, the asymptotical normal distribution of the MLE under standard regularity conditions holds. 


\subsection{Application 1: Skin Folds Data}

First, we consider the data discussed in Weisberg (2005, Section 6.4), which represent 102 male and 100 female athletes collected at the Australian Institute of Sport, courtesy of Richard Telford and Ross Cunningham. The following variable is evaluated in this study: sum of skin folds (skin folds). We compare the fits of the McBXII distribution with those of the BBXII, KwBXII and BXII distributions to these data.

In the last few years, several extensions of the BXII distribution have been introduced in the literature. For example, Silva and Cordeiro (2013) proposed the Burr XII power series distributions and studied some of their mathematical properties including moments, quantile and generating functions, order statistics and their moments, Kullback-Leibler divergence and Shannon entropy. They investigated two models called the Burr XII Poisson (BXIIP) and Burr XII geometric (BXIIG) distributions. The pdf and cdf of the BXIIP and BXIIG models are given, respectively, by

\section{- BXIIP distribution}

$$
f(x ; c, \lambda, k)=\frac{\lambda c k}{[\exp (\lambda)-1]} x^{c-1}\left(1+x^{c}\right)^{-k-1} \exp \left[\lambda\left(1+x^{c}\right)^{-k}\right], \quad x>0
$$

and

$$
F(x ; c, \lambda, k)=1-\frac{\exp \left[\lambda\left(1+x^{c}\right)^{-k}\right]-1}{[\exp (\lambda)-1]}, \quad x>0,
$$

where $\lambda>0, c>0$ and $k>0$.

\section{- BXIIG distribution}

$$
f(x ; \lambda, c, k)=\frac{c k(1-\lambda) x^{c-1}\left(1+x^{c}\right)^{-k-1}}{\left[1-\lambda\left(1+x^{c}\right)^{-k}\right]^{2}}, \quad x>0
$$

and

$$
F(x ; c, \lambda, k)=\frac{1-\left(1+x^{c}\right)^{-k}}{1-\lambda\left(1+x^{c}\right)^{-k}}, \quad x>0,
$$

where $0<\lambda<1, c>0$ and $k>0$.

Initial values for $s, k$ and $\alpha$ are taken from the fitted BXII model with $a=b=c=1$; see, for example, Silva et al. (2008). The computations are performed using the subroutine NLMIXED in SAS. Table 2 lists the MLEs (and the corresponding standard errors in parentheses) of the model parameters and the values of the following statistics for some fitted models: Akaike Information Criterion (AIC), Consistent Akaike Information Criterion (CAIC) and Bayesian Information Criterion (BIC). The computations are done using the NLMixed subroutine in SAS. These results indicate that the McBXII model has the lowest AIC, CAIC and BIC values among those values of the fitted models, and therefore it could be chosen as the best model.

A comparison of the proposed distribution with some of its sub-models using LR statistics is performed in Table 3. The figures in this table, specially the p-values, indicate that the McBXII model gives a better fit to these data than the other three sub-models.

More information is provided by a visual comparison of the histogram of the data with the fitted density functions. The plots of the fitted McBXII, BNXII, KwBXII, BXII, BXIIP and BXIIG densities are displayed in Figures 5 and 6 for the skin folds data. Clearly, the McBXII distribution provides a closer fit to the histogram than the other three sub-models.

\subsection{Application 2: Data of Animal Science with Censored Data}

The data come from the zootechnics records of a Brazilian company engaged in raising beef cattle, where the farms stocked with the Nelore breed are located in the states of Bahia and São Paulo. In the analysis, only data on females born in 2,000 are used and the age at first calving is the reproductive characteristic analyzed. In this case, the response variable $\left(y_{i}\right)$ is the logarithm of the age of the cows at first calving (measured in days). The first calving age is an important characteristic for beef cattle breeders because the faster cows reach reproductive maturity, the more calves they will produce during their breeding cycle and the greater the breeder's return on investment will be. Further, this trait is 
Table 2. Estimates of the model parameters for the skin folds data, the corresponding SEs (given in parentheses) and the AIC, CAIC and BIC statistics.

\begin{tabular}{|c|c|c|c|c|c|c|c|c|c|}
\hline Model & $a$ & $b$ & $c$ & $s$ & $k$ & $\alpha$ & $\overline{\mathrm{AIC}}$ & CAIC & $\overline{\mathrm{BIC}}$ \\
\hline$\overline{\mathrm{McBXII}}$ & $\begin{array}{c}0.0680 \\
(0.0051)\end{array}$ & $\begin{array}{l}20.7921 \\
(0.7563)\end{array}$ & $\begin{array}{l}32.6303 \\
(4.3666)\end{array}$ & $\begin{array}{l}26.9624 \\
(1.8218)\end{array}$ & $\begin{array}{c}0.0359 \\
(0.0485)\end{array}$ & $\begin{array}{l}37.7378 \\
(8.3168)\end{array}$ & 1901.0 & $\overline{1901.4}$ & 1920.8 \\
\hline BBXII & $\begin{array}{l}31.0689 \\
(0.2739)\end{array}$ & $\begin{array}{c}1.0627 \\
(0.1348)\end{array}$ & $\begin{array}{l}1 \\
-\end{array}$ & $\begin{array}{l}38.7013 \\
(7.2524)\end{array}$ & $\begin{array}{c}3.9215 \\
(3.1056)\end{array}$ & $\begin{array}{c}1.0576 \\
(0.3960)\end{array}$ & 1919.7 & 1920.0 & 1936.3 \\
\hline KwBXII & $\begin{array}{l}1 \\
-\end{array}$ & $\begin{array}{c}0.1643 \\
(0.0122)\end{array}$ & $\begin{array}{l}1514.94 \\
(110.64)\end{array}$ & $\begin{array}{c}10064.0 \\
(4248.62)\end{array}$ & $\begin{array}{l}1086.94 \\
(192.89)\end{array}$ & $\begin{array}{c}0.8795 \\
(0.0644)\end{array}$ & 1908.4 & 1908.7 & 1924.9 \\
\hline BXII & $\begin{array}{l}1 \\
-\end{array}$ & $\begin{array}{l}1 \\
-\end{array}$ & $\begin{array}{l}1 \\
-\end{array}$ & $\begin{array}{l}47.3262 \\
(5.8414)\end{array}$ & $\begin{array}{c}0.4884 \\
(0.1919)\end{array}$ & $\begin{array}{c}5.3015 \\
(1.1738)\end{array}$ & 1929.2 & 1929.3 & 1939.1 \\
\hline Model & & & $\bar{c}$ & $\lambda$ & $\bar{k}$ & & $\overline{\mathrm{AIC}}$ & $\overline{\mathrm{CAIC}}$ & $\overline{\mathrm{BIC}}$ \\
\hline BXIIP & & & $\begin{array}{c}3.4211 \\
(1.6784)\end{array}$ & $\begin{array}{l}15.6556 \\
(5.5822)\end{array}$ & $\begin{array}{l}0.00457 \\
(0.0011)\end{array}$ & & 2666.8 & 2666.9 & 2676.7 \\
\hline$\overline{\text { GXIIP }}$ & & & $\begin{array}{c}3.1639 \\
(7.0862)\end{array}$ & $\begin{array}{c}0.001 \\
(0.1621)\end{array}$ & $\begin{array}{c}0.0764 \\
(0.1712)\end{array}$ & & 2653.8 & 2653.9 & 2663.8 \\
\hline
\end{tabular}

Table 3. LR statistics for the skin folds data.

\begin{tabular}{c|c|c|c}
\hline \hline Model & Hypotheses & Statistic $\mathrm{w}$ & $p$-value \\
\hline \hline McBXII vs BBXII & $H_{0}: c=1$ vs $H_{1}: H_{0}$ is false & 20.7 & $<0.00001$ \\
McBXII vs KwBXII & $H_{0}: a=1$ vs $H_{1}: H_{0}$ is false & 9.4 & 0.0022 \\
McBXII vs BXII & $H_{0}: a=b=c=1$ vs $H_{1}: H_{0}$ is false & 34.2 & $<0.00001$ \\
\hline \hline
\end{tabular}

(a)

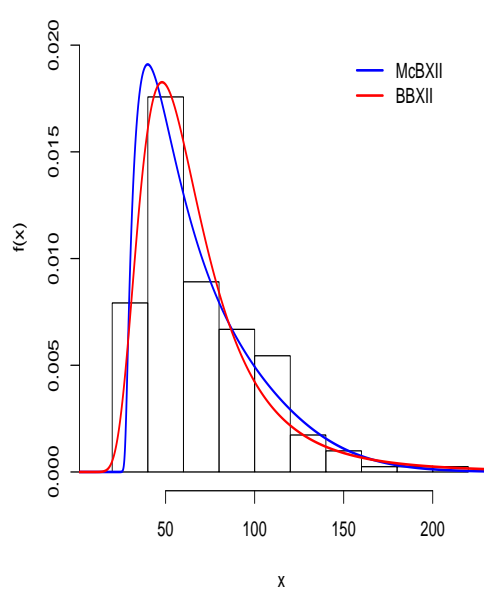

(b)

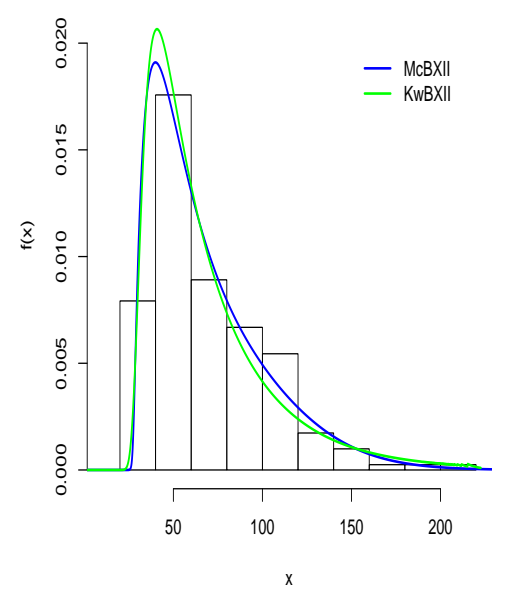

(c)

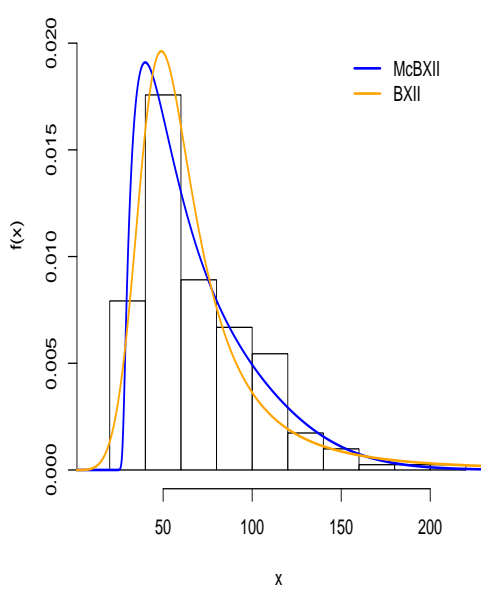

Figure 5. Fitted densities for the skin folds data. (a) Fitted McBXII vs BBXII models. (b) Fitted McBXII vs KwBXII models. (c) Fitted McBXII vs BXII models.

easy and inexpensive to measure. The sample size in this study is $n=1,326$, where $32.35 \%$ of the observations do not present the event of interest (calving) and are thus censored.

There are other factors that can also influence the age at first calving, such as the classification of cows at two levels-very young when the first calving age is less than or equal to 912 days and young when the age is greater than 912 days. In genetic assessments of Brazilian herds, the age at first calving is used without considering information on phenotype 
(a)

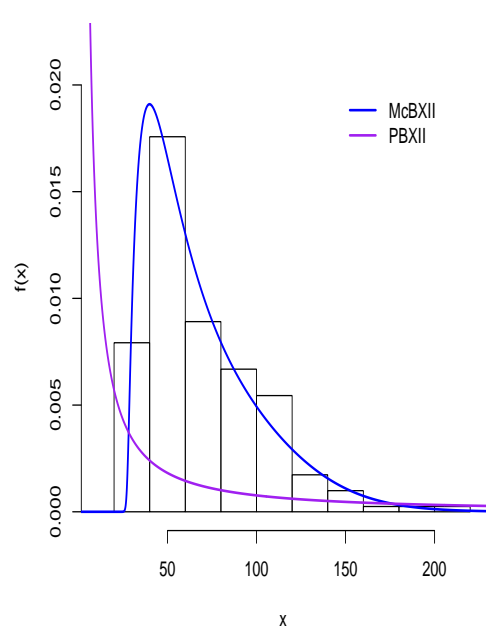

(b)

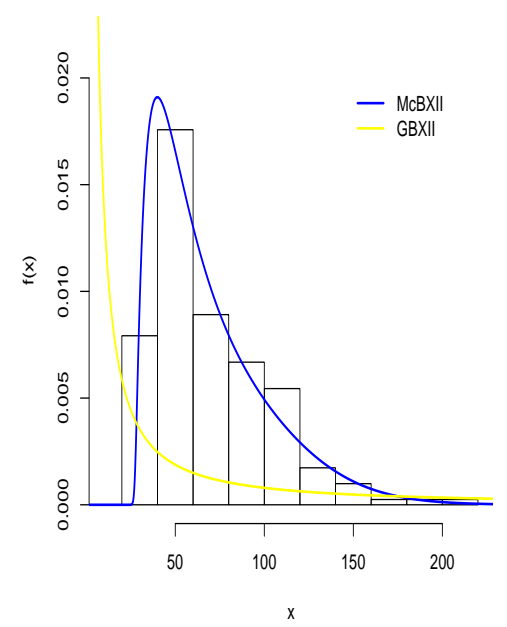

Figure 6. Fitted densities for the skin folds data. (a) Fitted McBXII vs PBXII models. (b) Fitted McBXII vs GBXII models.

(censoring) and without considering a suitable distribution to model these types of data. Thus, alternative approaches that consider censoring and heavy-tailed distributions are important. In this context, we consider the LMcBXII model and some of its special models to analyze these data. The variables considered in the models are: $y_{i}$ : logarithm of age of the cows at first calving, measured in days; $\delta_{i}$ : censoring indicator $(0=$ censoring, $1=$ time observed $)$ and $v_{i 1}$ : Age brackets ( 0 =young, 1 = very young).

We start the analysis of these data by considering only failure $\left(x_{i}\right)$ of the animal science data. An appropriate model for fitting these data could be the McBXII distribution. Initial values for $s, k$ and $\alpha$ are taken from the fitted BXII model with $a=b=c=1$. The computations are performed using the NLMIXED subroutine in SAS. Table 4 gives the MLEs (and the corresponding standard errors in parentheses) of the model parameters and the values of the AIC, BIC and CAIC statistics for some fitted models. These results indicate that the McBXII model has the lowest values of these statistics among those values of the fitted models, and therefore it could be chosen as the best model. Recently, various authors

Table 4. Estimates of the model parameters for the animal science data, the corresponding SEs (given in parentheses) and the AIC, CAIC and BIC statistics.

\begin{tabular}{c|cccccc|ccc}
\hline \hline Model & $a$ & $b$ & $c$ & $s$ & $k$ & $\alpha$ & AIC & CAIC & BIC \\
\hline \hline McBXII & 0.6078 & 0.4019 & 0.1104 & 1087.95 & 1.2492 & 89.5865 & 10755.0 & 10755.1 & 10783.0 \\
& $(0.0992)$ & $(0.0301)$ & $(0.0204)$ & $(3.7298)$ & $(0.0341)$ & $(0.9193)$ & & & \\
\hline BBXII & 0.1061 & 12.0154 & 1 & 1111.30 & 0.0290 & 78.5179 & 10779.0 & 10779.1 & 10803.0 \\
& $(0.0038)$ & $(0.1866)$ & - & $(2.8270)$ & $(0.0049)$ & $(0.5807)$ & & & \\
\hline KwBXII & 1 & 12.1802 & 0.1802 & 1210.67 & 0.0034 & 60.5037 & 10941.0 & 10941.1 & 10965.0 \\
& - & $(1.3172)$ & $(0.0058)$ & $(5.5396)$ & $(0.0001)$ & $(0.8184)$ & & & \\
\hline BXII & 1 & 1 & 1 & 1215.67 & 5.9503 & 11.68240 & 11033.0 & 11033.1 & 11048.0 \\
& - & - & - & $(23.5902)$ & $(1.0466)$ & $(0.3834)$ & & & \\
\hline \hline
\end{tabular}

studied properties and applications of the BW (Famoye et al., 2005) distribution in survival analysis and reliability. For example, Ortega et al. (2011) pioneered the log-beta Weibull regression model based on the BW distribution and Ortega et al. (2012) proposed the negative binomial-beta Weibull regression model to predict the cure fraction for patients with clinically localized prostate cancer treated by open radical prostatectomy. The BW distribution with parameters $a>0$, $b>0, \alpha>0$ and $\gamma>0$ has density function (for $x>0$ ) given by

$$
f(x)=\frac{\gamma(1 / \alpha)^{\gamma}}{B(a, b)} x^{\gamma-1} \exp \left\{-b(x / \alpha)^{\gamma}\right\}\left[1-\exp \left\{-(x / \alpha)^{\gamma}\right\}\right]^{a-1},
$$


where $a$ and $b$ are two additional shape parameters to the Weibull parameters to govern skewness and kurtosis.

An alternative approach for modeling these data can be provided by the Birnbaum-Saunders (BS) distribution. There are various extensions of this lifetime distribution. More recently, Cordeiro and Lemonte (2011) proposed the $\beta$-BirnbaumSaunders $(\beta$-BS) distribution for fatigue life modeling. They investigated various properties of the $\beta$-BS model including expansions for the moments, generating function, mean deviations, density function of the order statistics and their moments. The $\beta$-BS density function with four positive parameters $a, b, \alpha$ and $\beta$ is given by

$$
f(x)=\frac{\kappa(\alpha, \beta)}{B(a, b)} x^{-3 / 2}(x+\beta) \exp \left\{-\tau(x / \beta) /\left(2 \alpha^{2}\right)\right\} \Phi(v)^{a-1}\{1-\Phi(v)\}^{b-1}, \quad x>0,
$$

where $\kappa(\alpha, \beta)=\exp \left(\alpha^{-2}\right) /(2 \alpha \sqrt{2 \pi \beta}), \tau(z)=z+z^{-1}, v=\alpha^{-1} \rho(x / \beta), \rho(z)=z^{1 / 2}-z^{-1 / 2}$ and $\Phi(\cdot)$ is the standard normal cumulative distribution. For $a=b=1$, we obtain the BS distribution.

Table 5 provides the MLEs of the parameters (the standard errors are given in parentheses) for the BW, EW, $\beta$-BS and BS distributions fitted to the current data and the values of the AIC, CAIC and BIC statistics. By comparing the figures in Tables 1 and 2, we can verify that the McBXII distribution is a very competitive model to lifetime data. In fact, the McBXII distribution is a very good alternative model to the $\mathrm{BW}$ and $\beta$-BS distributions. In this application, it yields a better fit than the BW, EW, $\beta$-BS and BS distributions as shown by the AIC, CAIC and BIC values.

Table 5. MLEs for the BW, EW, $\beta$-BS and BS models and information criteria for the animal science data.

\begin{tabular}{c|cccc|ccc}
\hline \hline Model & $a$ & $b$ & $\alpha$ & $\gamma$ & AIC & CAIC & BIC \\
\hline \hline BW & 1.9612 & 2.2561 & 1086.99 & 6.7628 & 11078.0 & 11078.1 & 11098.0 \\
& $(0.3210)$ & $(1.3308)$ & $(78.1509)$ & $(0.6276)$ & & & \\
EW & 1.7310 & 1 & 997.84 & 7.3443 & 11080.0 & 11080.1 & 11094.0 \\
& $(0.2175)$ & - & $(14.7552)$ & $(0.4750)$ & & & \\
\hline \hline Model & $a$ & $b$ & $\alpha$ & $\beta$ & AIC & CAIC & BIC \\
\hline \hline$\beta$-BS & 0.1921 & 0.2157 & 0.04614 & 996.19 & 11105.0 & 11105.1 & 11124.0 \\
& $(0.0147)$ & $(0.0111)$ & $(0.00094)$ & $(7.3382)$ & & & \\
BS & 1 & 1 & 0.1218 & 996.92 & 11156.0 & 11157.0 & 11166.0 \\
& - & - & $(0.0029)$ & $(4.0466)$ & & & \\
\hline \hline
\end{tabular}

A comparison of the proposed distribution with some of its sub-models using LR statistics is done in Table 6. The figures in this table, specially the p-values, indicate that the McBXII model gives a better fit to these data than the other three sub-models.

Table 6. LR statistics for the animal science data.

\begin{tabular}{c|c|c|c}
\hline \hline Model & Hypotheses & Statistic $\mathrm{w}$ & $p$-value \\
\hline \hline McBXII vs BBXII & $H_{0}: c=1$ vs $H_{1}: H_{0}$ is false & 26.0 & $<0.00001$ \\
McBXII vs KwBXII & $H_{0}: a=1$ vs $H_{1}: H_{0}$ is false & 188.0 & $<0.00001$ \\
McBXII vs BXII & $H_{0}: a=b=c=1$ vs $H_{1}: H_{0}$ is false & 284.0 & $<0.00001$ \\
\hline \hline
\end{tabular}

Next, we present the results from the fit of the regression model

$$
y_{i}=\beta_{0}+\beta_{1} v_{i 1}+\sigma z_{i},
$$

where the random variable $z_{i}$ has the LMcBXII distribution (13) for $i=1, \ldots, 1326$. The MLEs of the model parameters given in Table 7 are evaluated using the NLMixed procedure in SAS. Iterative maximization of the logarithm of the likelihood function (15) starts with initial values for $\beta$ and $\sigma$ obtained from the fitted LBXII regression model with $a=b=c=1$; see Silva et al. (2008).

A summary of the values of the AIC, CAIC and BIC statistics to compare the LMcBXII, LBBXII, LKwBXII and LBXII regression models is given in Table 8. The LMcBXII regression model provides the best fit according to these criteria. 
Table 7. MLEs of the parameters from the LMCBXII regression model fitted to the animal science data, the corresponding SEs (given in parentheses) and p-values in [.].

\begin{tabular}{c|ccccccc}
\hline \hline Model & $\mathrm{a}$ & $\mathrm{b}$ & $\mathrm{c}$ & $\mathrm{k}$ & $\sigma$ & $\beta_{0}$ & $\beta_{1}$ \\
\hline \hline LMCBXII & 0.3070 & 0.6397 & 0.3955 & 0.0032 & 0.0053 & 6.9944 & -0.2523 \\
& $(0.1126)$ & $(0.1644)$ & $(0.1619)$ & $(0.0010)$ & $(0.00009)$ & $(0.0015)$ & $(0.0029)$ \\
& & & & & & {$[<0.0001]$} & {$[<0.0001]$} \\
\hline \hline
\end{tabular}

Table 8. The AIC, CAIC and BIC statistics for comparing the LMcBXII, LBBXII, LKwBXII and LBXII regression models.

\begin{tabular}{c|ccc}
\hline \hline Model & AIC & CAIC & BIC \\
\hline \hline LMcBXII & -1251.0 & -1250.0 & -1214.0 \\
LBBXII & -1227.1 & -1227.0 & -1196.0 \\
LKwBXII & -1218.1 & -1218.0 & -1187.0 \\
LBXII & -426.2 & -426.1 & -405.4 \\
\hline \hline
\end{tabular}

A comparison of the proposed distribution with some of its sub-models using LR statistics is performed in Table 9. The figures in this table, specially the p-values, indicate that the LMcBXII regression model provides a better fit to the current data than the other three distributions. We note from the fitted LMcBXII regression model that $v_{1}$ and $v_{2}$ are significant at $1 \%$ and that there is a significant difference between the levels of the covariate $v_{1}$. Note that this significance can only serve as a way to discover traits that differ between the two groups.

Table 9. LR statistics for the animal science data.

\begin{tabular}{c|c|c|c}
\hline \hline Model & Hypotheses & Statistics $\mathrm{w}$ & $P$-value \\
\hline \hline LMcBXII vs LBBXII & $H_{0}: c=1$ vs $H_{1}: H_{0}$ is false & 26.0 & $<0.00001$ \\
LMcBXII vs LKwBXII & $H_{0}: a=1$ vs $H_{1}: H_{0}$ is false & 35.0 & $<0.00001$ \\
LMcBXII vs LBXII & $H_{0}: a=b=c=1$ vs $H_{1}: H_{0}$ is false & 830.1 & $<0.00001$ \\
\hline \hline
\end{tabular}

We perform the residual analysis by plotting in Figure 7 the modified martingale-type residuals $r_{M D i}$ (see Section 8.1) against the index of the observations. We note no pattern of the residuals in the interval $(-2,2)$ and no outliers. In fact, there are two distinct groups: the first one consists of failure observations and the second one refers to the censored observations, where the censoring time occurs at the end of the study. They have the same residual value equal to -1.5305 .

\section{Concluding Remarks}

We propose a new six-parameter lifetime model called the McDonald-Burr XII distribution, which generalizes the betaBurr XII (Paranaíba et al., 2011), Kumaraswamy-Burr XII (Paranaíba et al., 2012), Kumaraswamy log-logistic, Kumaraswamy Weibull, exponentiated Burr XII, beta log-logistic, beta Pareto type II, beta Weibull, beta exponential and Weibull distributions. Due to its flexibility in accommodating the four possible forms of the hazard function, it is an important model for modeling lifetime data. The proposed model fits much better to positive real data than the Burr XII distribution since it involves three additional shape parameters. We provide a mathematical treatment of the proposed distribution including a useful expansion for its density function. We derive closed-form expressions for the moments and generating function. The model parameters are estimated by maximum likelihood.

Based on the McBXII distribution, we propose the log-McBurr XII regression model, which is suitable for modeling censored and uncensored lifetime data. The new regression model allows us to test the goodness of fit of some known regression models as special models and then it serves as a good alternative for lifetime data analysis. We present a residual analysis based on two types of residuals for studying departures from the error distribution. We show by means of two real data sets that the proposed models can give better fits than some of their sub-models. 


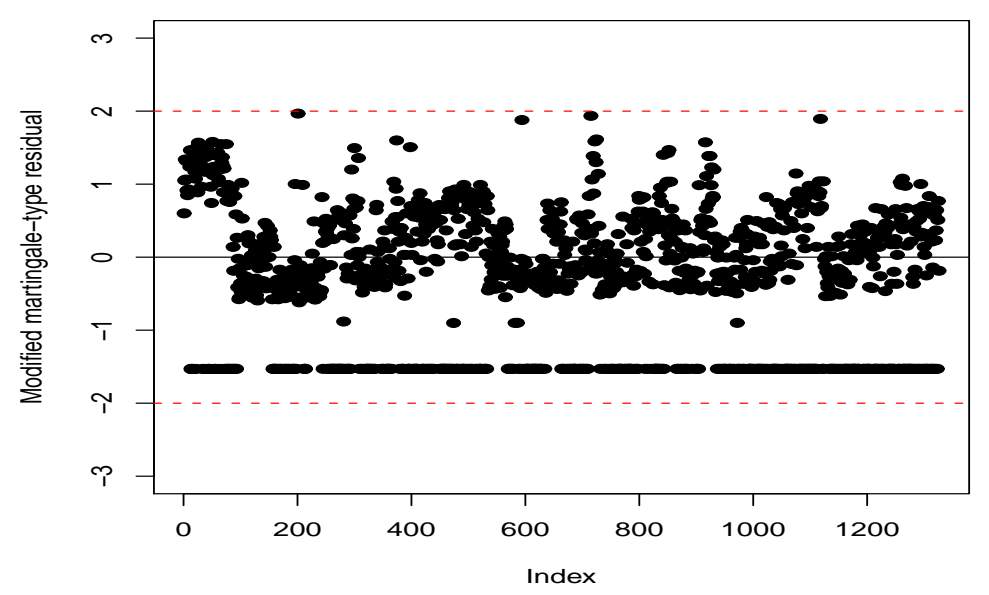

Figure 7. Index plot of the modified martingale-type residual for the the animal science data.

\section{Appendix A}

The elements of the score vector $U(\boldsymbol{\theta})$ for the parameters $(a, b, c, s . k, \alpha)$ are:

$$
\begin{aligned}
& U_{a}(\boldsymbol{\theta})=-r[\psi(a)-\psi(a+b)]+c \sum_{i \in F} \log \left(u_{i}\right)-\sum_{i \in D} \frac{\left[\dot{I}_{u_{i}^{c}}(a, b)\right]_{a}}{\left[1-I_{u_{i}^{c}}(a, b)\right]}, \\
& U_{b}(\boldsymbol{\theta})=-r[\psi(b)-\psi(a+b)]+\sum_{i \in F} \log \left(1-u_{i}^{c}\right)-\sum_{i \in D} \frac{\left[\dot{I}_{u_{i}^{c}}(a, b)\right]_{b}}{\left[1-I_{u_{i}^{c}}(a, b)\right]} \\
& U_{c}(\boldsymbol{\theta})=\frac{r}{c}+a \sum_{i \in F} \log \left(u_{i}\right)-(b-1) \sum_{i \in F} \frac{u_{i}^{c} \log \left(u_{i}\right)}{\left(1-u_{i}^{c}\right)}-\sum_{i \in D} \frac{\left[\dot{I}_{u_{i}^{c}}(a, b)\right]_{c}}{\left[1-I_{u_{i}^{c}}(a, b)\right]}, \\
& U_{s}(\boldsymbol{\theta})=-\frac{r \alpha}{s}-\frac{(k+1) \alpha}{s^{\alpha+1}} \sum_{i \in F} \frac{x_{i}^{\alpha}\left(1-u_{i}\right)^{\frac{k+1}{k}}}{\left(1-u_{i}\right)}-\frac{(a c-1) k \alpha}{s^{\alpha+1}} \sum_{i \in F} \frac{x_{i}^{\alpha}\left(1-u_{i}\right)^{\frac{k+1}{k}}}{u_{i}} \\
& +\frac{(b-1) c k \alpha}{s^{\alpha+1}} \sum_{i \in F} \frac{x_{i}^{\alpha} u_{i}^{c-1}\left(1-u_{i}\right)^{\frac{k+1}{k}}}{\left(1-u_{i}^{c}\right)}-\sum_{i \in D} \frac{\left[\dot{I}_{u_{i}^{c}}(a, b)\right]_{s}}{\left[1-I_{u_{i}^{c}}(a, b)\right]}, \\
& U_{k}(\boldsymbol{\theta})=\frac{r}{k}-\frac{1}{k} \sum_{i \in F} \log \left(1-u_{i}\right)-\frac{(a c-1)}{k} \sum_{i \in F} \frac{\left(1-u_{i}\right) \log \left(1-u_{i}\right)}{u_{i}} \\
& +\frac{(b-1) c}{k} \sum_{i \in F} \frac{u_{i}^{c-1}\left(1-u_{i}\right) \log \left(1-u_{i}\right)}{\left(1-u_{i}^{c}\right)}-\sum_{i \in D} \frac{\left[\dot{I}_{u_{i}^{c}}(a, b)\right]_{k}}{\left[1-I_{u_{i}^{c}}(a, b)\right]},
\end{aligned}
$$

and

$$
\begin{aligned}
U_{\alpha}(\boldsymbol{\theta})= & \frac{r}{\alpha}-r \log (s)-\frac{(k+1)}{s^{\alpha}} \sum_{i \in F} x_{i}^{\alpha}\left(1-u_{i}\right)^{1 / k} \log \left(x_{i} / s\right)+\sum_{i \in F} \log \left(x_{i}\right) \\
& +\frac{(a c-1) k}{s^{\alpha}} \sum_{i \in F} \frac{x_{i}^{\alpha}\left(1-u_{i}\right)^{\frac{k+1}{k}} \log \left(x_{i} / s\right)}{u_{i}}-\frac{(b-1) c k}{s^{\alpha}} \sum_{i \in F} \frac{x_{i}^{\alpha} u_{i}^{c-1}\left(1-u_{i}\right)^{\frac{k+1}{k}} \log \left(x_{i} / s\right)}{\left(1-u_{i}^{c}\right)} \\
& -\sum_{i \in D} \frac{\left[\dot{I}_{u_{i}^{c}}(a, b)\right]_{\alpha}}{\left[1-I_{u_{i}^{c}}(a, b)\right]},
\end{aligned}
$$


where $\psi(\cdot)$ is the digamma function and

$$
\begin{array}{lll}
{\left[\dot{I}_{u_{i}^{c}}(a, b)\right]_{a}=\frac{\partial\left[I_{u_{i}^{c}}(a, b)\right]}{\partial a},} & {\left[\dot{I}_{u_{i}^{c}}(a, b)\right]_{b}=\frac{\partial\left[I_{u_{i}^{c}}(a, b)\right]}{\partial b}} & {\left[\dot{I}_{u_{i}^{c}}(a, b)\right]_{c}=\frac{\partial\left[I_{u_{i}^{c}}(a, b)\right]}{\partial c}} \\
{\left[\dot{I}_{u_{i}^{c}}(a, b)\right]_{s}=\frac{\partial\left[I_{u_{i}^{c}}(a, b)\right]}{\partial s},} & {\left[\dot{I}_{u_{i}^{c}}(a, b)\right]_{k}=\frac{\partial\left[I_{u_{i}^{c}}(a, b)\right]}{\partial k},} & {\left[\dot{I}_{u_{i}^{c}}(a, b)\right]_{\alpha}=\frac{\partial\left[I_{u_{i}^{c}}(a, b)\right]}{\partial \alpha}}
\end{array}
$$

\section{Appendix B}

Theorem B.1. Let $(\Omega, \mathfrak{F}, \mathbf{P})$ be a given probability space and let $H=[a, b]$ be an interval for some $a<b(a=-\infty, b=$ $\infty$ might as well be allowed). Let $X: \Omega \rightarrow H$ be a continuous random variable with distribution function $F$ and let $q$ and $h$ be two real functions defined on $H$ such that

$$
E[q(X) \mid X \geq x]=E[h(X) \mid X \geq x] \eta(x), \quad x \in H,
$$

is defined for some real function $\eta$. Assume that $q, h \in C^{1}(H), \eta \in C^{2}(H)$ and $F$ is twice continuously differentiable and strictly monotone function on the set $H$. Further, we assume that the equation $h \eta=q$ has no real solution in the interior of $H$. Then, $F$ is uniquely determined by the functions $q, h$ and $\eta$, particularly

$$
F(x)=C \int_{a}^{x}\left|\frac{\eta^{\prime}(u)}{\eta(u) h(u)-q(u)}\right| \exp [-s(u)] d u,
$$

where $s=s(x)$ is a solution of the differential equation $s^{\prime}(x)=\left[\eta^{\prime}(x) h(x)\right] /[\eta(x) h(x)-q(x)]$ and $C$ is a constant chosen such that $\int_{H} d F=1$.

\section{Appendix C}

The SAS code for the NLMixed procedure to fit the McBXII model. More details on this procedure, see http://support.sas.com/documentation/onlinedoc/stat/930/nlmixed.pdf.

proc nlmixed cov data=listal;

parms $a=1 \quad b=1 \quad c=1 \quad s=1 \quad k=1$ alpha=1;

bounds $a>0$;

bounds $b>0$;

bounds $c>0$;

bounds $s>0$;

bounds $k>0$;

bounds alpha $>0$;

$f=$ alpha $* k *(s * *(-$ alpha $)) *(t * *($ alpha -1$)) *(1+(t / s) * *($ alpha $)) * *(-k-1)$;

$F F=1-(1+(t / s) * *($ alpha $)) * *(-k)$;

$f t=(c / \operatorname{Beta}(a, b)) * f^{*}\left((F F) * *\left(a^{*} c-1\right)\right) *((1-(F F) * * c) * *(b-1))$;

$s t=1-\operatorname{probbeta}\left((F F)^{* *} c, a, b\right)$;

$l v=\log (f t)$;

model t general $(l v)$;

run;

\section{References}

Alexander, C., Cordeiro, G. M., Ortega, E. M. M., \& Sarabia, J. M. (2012). Generalized beta-generated distributions. Computational Statistics and Data Analysis, 56, 1880-1897. http://dx.doi.org/10.1016/j.csda.2011.11.015

Cordeiro, G. M., \& de Castro, M. (2011). A new family of generalized distributions. Journal of Statistical Computation and Simulation, 81, 883-898. http://dx.doi.org/10.1080/00949650903530745

Cordeiro, G. M., \& Lemonte, A. J. (2011). The $\beta$-Birnbaum-Saunders distribution: An improved distribution for fatigue life modeling. Computational Statistics and Data Analysis, 55, 1445- 1461. http://dx.doi.org/10.1016/j.csda.2010.10.007

Doornik, J. (2007). An Object-oriented Matrix Programming Language Ox 5, 5th ed. Timberlake Consultants Press, London.

Eugene, N., Lee, C., \& Famoye, F. (2002). Beta-normal distribution and its applications. Communications in Statistics Theory and Methods, 31, 497-512. http://dx.doi.org/10.1081/STA-120003130 
Famoye, F., Lee, C., \& Olumolade, O. (2005). The beta-Weibull distribution. Journal of Statistical Theory and Applications, 4, 121-136.

Glänzel, W. (1987). A characterization theorem based on truncated moments and its application to some distribution families. Mathematical Statistics and Probability Theory (Bad Tatzmannsdorf, 1986) B, Reidel, Dordrecht, 75-84. http://dx.doi.org/10.1007/978-94-009-3965-3_8

Gradshteyn, I. S., \& Ryzhik, I. M. (2000). Table of Integrals, Series and Products (sixth edition). Academic Press: San Diego.

Hashimoto, E. M., Ortega, E. M. M., Cancho, V. G., \& Cordeiro, G. M. (2010). The log-exponentiated Weibull regression model for interval-censored data. Computational Statistics and Data Analysis, 54, 1017-1035. http://dx.doi.org/10.1016/j.csda.2009.10.014

Kenney, P. F., \& Keeping, E. S. (1962). Mathematics of statistics, 3rd edition. Van Nostrand: New Jersey.

Klein, J. P., \& Moeschberger, M. L. (1997). Survival Analysis: Techniques for Censored and Truncated Data. Springer: New York. http://dx.doi.org/10.1007/978-1-4757-2728-9

Kumaraswamy, P. (1980). Generalized probability density-function for double-bounded random- processes. Journal of Hydrology, 46, 79-88. http://dx.doi.org/10.1016/0022-1694(80)90036-0

Lawless, J. F. (2003). Statistical Models and Methods for Lifetime Data. Wiley: New York.

McCullagh, P., \& Nelder, J. A. (1989). Generalized Linear Models. 2nd ed. Chapman and Hall: London. http://dx.doi.org/10.1007/978-1-4899-3242-6

Molenberghs, G., Verbeke, G., Demétrio, C. G. B. and Vieira, A. M.C. (2010). A family of generalized linear models for repeated measures with normal and conjugate random effects. Statistical Science, 25, 325-347. http://dx.doi.org/10.1214/10-STS328

Moors, J. J. A. (1998). A quantile alternative for kurtosis. Journal of the Royal Statistical Society Serie D, The Statistician, 37, 25-32. http://dx.doi.org/10.2307/2348376

Mudholkar, G. S., Srivastava, D. K., \& Friemer, M. (1995). The exponentiated Weibull family: A reanalysis of the bus-motor-failure data. Technometrics, 37, 436-445. http://dx.doi.org/10.1080/00401706.1995.10484376

Mudholkar, G. S., Srivastava, D. K., \& Kollia, G. D. (1996) A generalization of the Weibull distribution with application to the analysis of survival data. Journal American Statical Association, 91, 1575-1583. http://dx.doi.org/10.1080/01621459.1996.10476725

Nadarajah, S., \& Gupta, A. K. (2007). A generalized gamma distribution with application to drought data. Journal Mathematics and Computers in Simulation, 74, 1-7. http://dx.doi.org/10.1016/j.matcom.2006.04.004

Ortega, E. M. M., Paula, G. A., \& Bolfarine, H. (2008). Deviance residuals in generalized log-gamma regression models with censored observations. Journal of Statistical Computation and Simulation, 78, 747-764. http://dx.doi.org/10.1080/00949650701282465

Ortega, E. M. M., Cordeiro, G. M., \& Hashimoto, E. M. (2011). A log-linear regression model for the beta-Weibull distribution. Communications in Statistics-Simulation and Computation, 40, 1206-1235. http://dx.doi.org/10.1080/03610918.2011.568150

Ortega, E. M. M., Cordeiro, G. M., \& Kattan, M. W. (2012). The negative binomial-beta Weibull regression model to predict the cure of prostate cancer. Journal of Applied Statistics, 39, 1191-1210. http://dx.doi.org/10.1080/02664763.2011.644525

Paranaíba, F. P., Ortega, E. M. M., Cordeiro, G. M., \& Pescim, R. R. (2011). The beta Burr XII distribution with application to lifetime data. Computational Statistics and Data Analysis, 55, 1118-1136. http://dx.doi.org/10.1016/j.csda.2010.09.009

Paranaíba, F. P., Ortega, E. M. M., Cordeiro, G. M., \& Pascoa, M. A. R. (2012). The Kumaraswamy Burr XII Distribution: Theory and Practice. Journal of Statistical Computation and Simulation. http://dx.doi.org/10.1080/00949655.2012.683003

Silva, G. O., Ortega, E. M. M., Garibay, V. C., \& Barreto, M. L. (2008). Log-Burr XII regression models with censored data. Computational Statistics and Data Analysis, 52, 3820-3842. http://dx.doi.org/10.1016/j.csda.2008.01.003

Silva, G. O., Ortega, E. M. M., \& Paula, G. A. (2011). Residuals for log-Burr XII regression models in survival analysis. 
Journal of Applied and Statistics, 38, 1435-1445. http://dx.doi.org/10.1080/02664763.2010.505950

Silva, R. B., \& Cordeiro, G. M. (2013). The Burr XII power series distributions: A new compounding family. Statistics. In Press.

Therneau, T. M., Grambsch, P. M., \& Fleming, T. R. (1990). Martingale-based residuals for survival models. Biometrika, 77, 147-160. http://dx.doi.org/10.1093/biomet/77.1.147

Vangeneugden, T., Molenberghs, G., Verbeke, G., \& Demétrio, C. G. B. (2011). Marginal correlation from an extended random-effects model for repeated and overdispersed counts. Journal of Applied Statistics, 38, 215-232. http://dx.doi.org/10.1080/02664760903406405

Weisberg, S. (2005). Applied linear regression (3rd ed.). Wiley: New York. http://dx.doi.org/10.1002/0471704091

\section{Copyrights}

Copyright for this article is retained by the author(s), with first publication rights granted to the journal.

This is an open-access article distributed under the terms and conditions of the Creative Commons Attribution license (http://creativecommons.org/licenses/by/3.0/). 